\title{
Resección o trasplante en el tratamiento del carcinoma hepatocelular
}

\author{
L. Vázquez Velasco, L. Barneo Sierra
}

El carcinoma hepatocelular $(\mathrm{CHC})$ representa más del $90 \%$ de las neoplasias hepáticas primarias y es un tumor de amplia distribución en todo el mundo con una característica variabilidad geográfica en su incidencia ${ }^{1}$. Se estima que es responsable de un millón de muertes anuales ${ }^{2}$. Tradicionalmente, se ha considerado su distribución mundial en zonas de baja, media y alta incidencia, perteneciendo nuestro país a un área de incidencia baja/media (menos de 20 casos nuevos por 100.000 habitantes y año). Sin embargo, cada vez más informes reflejan el incremento creciente del $\mathrm{CHC}$ en el mundo occidenta $^{3-5}$ principalmente en relación con el aumento de la prevalencia de las hepatopatías por virus $C$ de la hepatitis.

Estos aspectos epidemiológicos y otros relacionados con la historia natural de carcinoma hepatocelular, son muy relevantes a la hora de planear estrategias terapéuticas. Sólo un 10 a $15 \%$ de los $\mathrm{CHC}$ se originan sobre hígados sanos ${ }^{6}$. Hasta un $90 \%$ de nuestros pacientes con $\mathrm{CHC}$ tendrán una hepatopatía subyacente, principalmente cirrosis post-hepatitis, por abuso de alcohol o hemocromatosis, lo que les confiere otra dimensión como pacientes además de la estrictamente oncológica. De otra parte, el $\mathrm{CHC}$ sobre un hígado cirrótico es, con frecuencia, multifocal a la hora del diagnóstico y es asimismo frecuente la aparición de tumores "de novo" en el seguimiento de pacientes a quienes se trató con éxito un primer tumor.

La historia natural del $\mathrm{CHC}$, tanto en áreas endémicas como en el mundo occidental, es muy desfavorable. La media de supervivencia de los pacientes no tratados está en torno a cuatro meses ${ }^{7}$.

Hasta hace poco más de una década, la resección hepática era considerada el único tratamiento potencialmente curativo del $\mathrm{CHC}$ sobre hígado cirrótico, pero el tratamiento debía limitarse a pacientes con una función hepática suficientemente buena para sobrevivir a la cirugía y a la merma funcional resultante de la pérdida de parénquima hepático

Unidad de Cirugía Hepato-bilio-pancreática y trasplante Servicio de Cirugía I

Hospital Universitario Central de Asturias tras la extirpación. De no hacerlo así, la mortalidad operatoria o por insuficiencia hepática en el seguimiento, resultarían prohibitivas. Esto restringe de modo muy importante la aplicabilidad del procedimiento.

La resección del $\mathrm{CHC}$ acredita supervivencias a cinco años entre el 30 y $40 \% 8,9$, con una llamativa disparidad en indicaciones, operabilidad y resultados entre los autores de centros orientales y occidentales y se ha sugerido que el $\mathrm{CHC}$ en las diferentes regiones del mundo podría representar formas diferentes de la enfermedad; sin embargo, esto no ha podido ser justificado de modo evidente ${ }^{10}$.

La irrupción del trasplante hepático como arma terapéutica segura en las hepatopatías crónicas terminales, abrió un nuevo escenario en la terapéutica del CHC. La hepatectomía total seguida de trasplante de un nuevo hígado sano, no sólo permitía eliminar por completo el tumor conocido sino también otros focos tumorales metacrónicos no diagnosticados, al tiempo que restauraría la función hepática a la normalidad. Sin embargo, los resultados de las primeras series no avalaron el entusiasmo despertado por la llegada del trasplante. Salvo en el llamado "hepatoma incidental"11, la supervivencia a cinco años era inferior al $40 \%$ y las tasas de recurrencia superaban el $50 \%{ }^{12}$. Como se comentará más adelante, estos primeros resultados fueron producto de una indicación liberal del trasplante sin tener en cuenta el estadio de la enfermedad tumoral. A día de hoy y cuando se cumplen los criterios de selección consensuados, el trasplante hepático es la mejor opción terapéutica para un paciente cirrótico con $\mathrm{CHC}$.

En estos últimos años se han introducido otros procedimientos terapéuticos no quirúrgicos, alcoholización ${ }^{13}$ y ablación por radiofrecuencia ${ }^{14}$ fundamentalmente, que no son objeto de esta revisión pero que acreditan excelentes resultados cuando se utilizan de acuerdo a las indicaciones y restricciones establecidas para su uso, y que principalmente vienen determinadas por el número, tamaño y localización de las lesiones.

\section{Determinantes para la elección de la terapéutica con intención curativa}

Ya se ha señalado que sólo de un 10 a 15\% de los pacientes con $\mathrm{CHC}$ tendrán un hígado "sano". Estos pacientes tendrán en su mayoría un tumor único, de gran tamaño y 
usualmente asintomático. La primera opción terapéutica a considerar es la resección quirúrgica. Parten de una función hepática norma, lo que permitirá resecciones masivas de hasta el 70 u $80 \%$ del parénquima hepático si ello fuera necesario. Únicamente la evidencia de enfermedad metastásica intra o extrahepática o la limitación técnica a la resección por imposibilidad de preservar el flujo venoso suprahepático o el pedicuro vásculo-biliar del hígado restante deben hacernos desistir del intento de resección.

El resto de los pacientes, hasta el $90 \%$, tendrán una cirrosis hepática de la que será preciso conocer su etiología (virus, alcohol, etc.) y el estatus del paciente en el momento de diagnóstico (actividad del virus, alcoholismo o abstinencia, etc.). Pero, de modo fundamental, debemos conocer la reserva funcional hepática y el "estado de salud" del paciente. La resección menor útil con pretensión curativa del $\mathrm{CHC}$, es la segmentectomía anatómica y debemos asegurarnos de que la pérdida de parénquima no deje al paciente en una insuficiencia hepática fatal.

La evaluación de estas variables no es fácil. Nos parece útil a este respecto el estadiaje según los criterios $\mathrm{BCLC}^{15}$ (Barcelona-Clinic Liver Cancer) que toman en cuenta el estatus funcional del paciente, el estatus de la hepatopatía y las características del tumor.

Obviamente, debe estar establecido que la lesión focal hepática que estamos evaluando es un $\mathrm{CHC}$. Esta certeza se obtiene por confirmación histológica mediante PAAF o biopsia o por criterios radiológicos (confirmación por dos técnicas de imagen de una lesión hipervascular mayor de dos centímetros). La, en principio, deseable confirmación citológica o histológica es cuestionable en el $\mathrm{CHC}$ sobre cirrosis. Aunque el riesgo es probablemente muy bajo $(1,6$ a $5,1 \%$ comunicado en la literatura), es posible la diseminación peritoneal o el implante del tumor en el trayecto de punción. Por otra parte, muchas veces no será posible puncionar todos o alguno de los nódulos detectados en los estudios de imagen. Por estas razones, muchos grupos renuncian a la prueba histológica.

Renunciar a la confirmación histológica tiene la servidumbre de incluir en tratamiento a pacientes con lesiones focales de naturaleza distinta al CHC. Los falsos positivos en el diagnóstico de $\mathrm{CHC}$, pueden tener un impacto negativo sobre el reparto de órganos de donantes entre los pacientes en lista de espera para trasplante, cuando se incluyen pacientes con diagnóstico de $\mathrm{CHC}$ que no serían candidatos a trasplante si atendiéramos únicamente al estado de su hepatopatía cróni$\mathrm{ca}^{16}$.

El número, localización sobre la anatomía hepática y la "masa" tumoral son las características fundamentales a determinar, y su peso en la decisión es conjunto. Un tumor único y pequeño es probablemente ideal para la opción de resección, pero su localización puede hacer previsible una cirugía compleja y de alto riesgo, lo que junto con otros determinantes puede orientar la terapéutica hacia al trasplante como mejor opción. Las localizaciones en los segmentos I, IVa y VIII deben considerarse localizaciones técnicamente complejas.

El tamaño tumoral tiene una influencia significativa sobre el fracaso terapéutico, tanto en el trasplante como en la resección ${ }^{17}$; en los casos de multiplicidad debe considerarse el tamaño acumulado de todos los nódulos. El tamaño tumoral obtenido por TAC en la evaluación preoperatoria se correla- ciona con la progresión del tumor, la invasión vascular y el índice de recurrencia después del trasplante.

Otro determinante pronóstico es la invasión vascular tanto macro como microscópica. La invasión vascular macroscópica debe ser establecida en el estudio preoperatorio y es un criterio de exclusión para tratamiento con intención curativa. La invasión vascular microscópica es, por ahora, un dato postoperatorio obtenido del estudio de la pieza de resección o del explante. Este hallazgo, junto con el tamaño tumoral mayor de 5 centímetros son predictores independientes de recurrencia tras tratamiento de intención curativa.

La cirugía del paciente cirrótico con $\mathrm{CHC}$ requiere de equipos profesionales expertos y tecnología sofisticada de diagnóstico y tratamiento. Parece por ello razonable que sea una patología regionalizada donde un grupo de hepatólogos, cirujanos, anestesistas, anatomopatólogos, radiólogos intervencionistas, etc., trabajen de modo continuado y conjunto sobre estos pacientes y puedan utilizar todos los recursos diagnósticos y de tratamiento disponibles. Pero la opción terapéutica a elegir no dependerá sólo de que la infraestructura y el equipo humano sean adecuados. La gran limitación del trasplante es la disponibilidad de órganos. Un paciente de buen pronóstico con resección o trasplante puede perder su oportunidad de curación si se elige la opción trasplante y se incluye en una lista de trasplante con un tiempo de espera largo ${ }^{18}$. Algunos países han decidido "primar" la posición en la lista de espera si el motivo de inclusión es un $\mathrm{CHC}$, no ya por el riesgo de muerte en lista, sino por el riesgo de progresión de la enfermedad a estadios no curables ${ }^{19}$.

\section{El debate resección versus trasplante}

No existen estudios controlados que comparen pacientes y tumores de las mismas características sometidos a resección o trasplante. Necesariamente, hemos de evaluar experiencias retrospectivas o prospectivas de ambos procedimientos pero no estudios comparativos.

Un problema común a cualquiera de ambos procedimientos es el conocimiento preoperatorio preciso de la extensión de la enfermedad. Las mayores dificultades se encuentran en determinar la naturaleza tumoral o no de los nódulos de tamaño inferior a dos centímetros y en las características microscópicas con trascendencia pronóstica como la invasión microvascular, la satelitosis, etc.

La primera limitación de la resección es la reserva hepática funcional. Incluso en pacientes en estadio A de Child, la descompensación hepática postoperatoria puede alcanzar al $50 \%$ de los pacientes. La presencia o no de hipertensión portal parece ser el mejor determinante del curso postoperatorio $^{20}$. La necesidad de realizar una resección lobar o multisegmentaria para asegurar la completa y oncológicamente correcta eliminación del tumor, contribuyen a agravar el riesgo de insuficiencia hepática postoperatoria.

El candidato "ideal" para resección es el paciente en estadio A de Child, sin ictericia ni hipertensión portal, que tiene un tumor único, menor de cinco centímetros y localizado en un segmento hepático periférico. En este grupo minoritario de pacientes con $\mathrm{CHC}$, cabe esperar una supervivencia e intervalo libre de enfermedad similares a los obtenidos con el trasplante y algunos autores han propuesto este procedimiento como tratamiento estándar para estos pacientes ${ }^{21}$. 


\section{Vázquez Velasco y L. Barneo Sierra}

La ventaja de la resección es la disponibilidad inmediata del procedimiento sin exponer al paciente a un tiempo de espera en lista, que en algunos grupos puede llegar a un año. El inconveniente es que la hepatopatía acompañante seguirá su evolución hacia la insuficiencia hepática y la casi inevitable recurrencia, predominantemente en el hígado restante. Esto ha llevado a algunos autores a proponer el trasplante como la mejor opción, incluso en los pacientes potencialmente resecables 22

En los últimos años ha surgido una postura intermedia que utiliza la resección como "puente" al trasplante ${ }^{23}$. Se propone resecar a aquellos pacientes en que ello sea posible aun cuando fueran elegibles para trasplante y reservar el trasplante para los que desarrollan recurrencia tumoral o progresión del deterioro de la función hepática. Quienes proponen esta estrategia se basan en que una vez ocurrida la recurrencia, el $79 \%$ de los pacientes son trasplantables con los mismos criterios que si se tratara de un trasplante primario.

Se ha valorado el impacto de una resección previa sobre la morbimortalidad del trasplante de rescate. Para algunos autores $^{24}$ la resección previa al trasplante hepático no incrementa la morbilidad ni altera la supervivencia a largo plazo del trasplante. Es cierto, sin embargo, que estos mismos autores comunican una mayor numero de infecciones, sangrado operatorio y reintervenciones que en los pacientes con trasplante primario. Otros grupos consideran que no más del $20 \%$, de los pacientes que hacen recurrencia o tienen progresión de su enfermedad hepática previa, serán trasplantables en esta estrategia de rescate y que la mortalidad operatoria es netamente superior a la de un trasplante primario ${ }^{25}$. Concluyen que la resección previa al trasplante disminuye claramente la opción de trasplante y las expectativas de supervivencia de los pacientes cirróticos con $\mathrm{CHC}$. Para estos autores, el trasplante es la primera opción terapéutica de estos pacientes, incluso cuando tienen un tumor resecable.

En este momento se esta considerando si los procedimientos ablativos, radiofrecuencia fundamentalmente, podrían sustituir a la resección en esta estrategia de "procedimiento puente" hasta el trasplante. Habría que demostrar que la ablación percutánea ejerce el mismo control de la enfermedad que la resección. De ser así, la opción seria muy interesante ya que evitaría al paciente los problemas derivados de una cirugía hepática previa al trasplante y su aplicabilidad, por lo que atañe al grado de insuficiencia hepática, se ampliaría a un mayor numero de pacientes.

Otra cuestión suscitada por la resección del $\mathrm{CHC}$ es que el examen anatomopatológico de la pieza permite identificar a aquellos pacientes de alto riesgo de recurrencia. Algunos autores proponen que la resección sirva para seleccionar el grupo de pacientes que debe ser dirigido a un procedimiento de trasplante sin demora. Deberíamos saber si los pacientes con una intervención resectiva oncologicamente correcta, que presentan un tumor de mal pronostico, tendrían mejores expectativas de supervivencia y de intervalo libre de enfermedad con un trasplante precoz de segunda intención. Esta cuestión permanece abierta por el momento.

Hasta Diciembre de 2.001 casi cincuenta mil pacientes han recibido un trasplante hepático en Europa ${ }^{26}$. Antes de 1980, el cáncer alcanzaba el 50\% de las indicaciones de trasplante hepático mientras que actualmente son solo el $10 \%$. El CHC supone el $8 \%$ de todas las indicaciones actua- les recogidas en el Registro Europeo de Trasplante hepático. Esta disminución relativa de la indicación de trasplante por cáncer se acompaña de una mejoría en la supervivencia desde el $11 \%$ a cinco años, en el periodo anterior a 1.985, al $59 \%$ en los últimos cinco años recogidos. Para el $\mathrm{CHC}$ en concreto, la supervivencia a 5 años se incrementa en un $17 \%$ entre 1.990 y 2.000 .

El cambio en los criterios de indicación y la mejoría en los resultados se produce cuando la comunidad del trasplante adopta los criterios propuestos por Bismuth (11) y popularizados por Mazzafero" ${ }^{27}$, conocidos como los "criterios de Milan" (ausencia de invasión vascular, nódulo único menor o igual a cinco centímetros de diámetro máximo o dos o tres nódulos de no mas de tres centímetros de diámetro conjunto). Existe hasta ahora el consenso de que estos pacientes pueden ser trasplantados con esperanza de obtener los mejores resultados posibles, similares a otras indicaciones de trasplante por enfermedad no neoplásica, entorno al $70 \%$ de supervivencia a cinco años. La asociación entre numero de nódulos y tamaño conjunto parece ser pues el mejor criterio predictivo de supervivencia.

La estimación del numero de nódulos y su tamaño se hace por los estudios de imagen preoperatorios. Por tanto, esta influenciada por la mejora en la resolución de las técnicas radiológicas que cada vez nos permiten "ver" más lesiones con las nuevas generaciones de TC lo que disminuirá el "infraestadiaje" actual. El impacto que esto tenga sobre los criterios de selección de pacientes para trasplante esta aun por determinar.

Aunque, como ya se ha dicho, los criterios de Milán son generalmente aceptados, se producen continuas comunicaciones de resultados variando el numero de nódulos admitido, el diámetro máximo de un nódulo único o el diámetro conjunto de varios nódulos. La limitación que impone la obtención de órganos para trasplante y los resultados de otros procedimientos alternativos hace fijar en $50 \%$ de supervivencia a cinco años el mínimo exigible para cualquier variación en los criterios de selección ${ }^{28}$.

La llegada del trasplante hepático de donante vivo parece convertirse en una buena alternativa al trasplante de cadáver. No existen aun series con numero y seguimiento suficiente para evaluar adecuadamente los resultados en trasplante por $\mathrm{CHC}$. Se ha establecido que existe un beneficio para el receptor cuando el tiempo en lista de espera para un trasplante convencional excede de siete meses. Si se obvia la discusión sobre los aspectos éticos del procedimiento y la morbi-mortalidad del donante, esta por definir si deben aceptarse las indicaciones con criterios expandidos, menos restrictivos que en el trasplante de donante cadáver.

Finalmente, parece necesario tener una actitud práctica para optar por resección o trasplante teniendo en cuenta todas la reflexiones que hasta aquí hemos hecho. Es cierto que nuestros algoritmos terapéuticos deben ser revisables a la luz de las aportaciones relevantes que se vayan produciendo pero, por ahora, podemos asumir que los pacientes en estadio A de Child, sin ictericia y sin hipertensión portal con un tumor resecable con bajo riesgo operatorio deben ser resecados, especialmente si la lista de espera para trasplante es superior a seis meses. Los pacientes no resecables por lo avanzado de su hepatopatía o la dificultad técnica de la resección, deben ser candidatos a trasplante si cumplen los criterios de Milán. De no cumplirlos, si se opta por el trasplan- 
te, deberán estar dentro de estudios controlados donde se realice una valoración critica de los resultados que, en todo caso, deben ofrecer al menos el $50 \%$ de supervivencia a cinco años.

\section{Bibliografía}

1. Rustgi VK. Epidemiology of hepatocellular carcinoma. Gastroenterol Clin N Am 1987;16:545-551.

2. Befeler AS, Di Bisceglie AM. Hepatocellular carcinoma : diagnosis and treatment. Gastroenterology. 2002;122:1609-1619

3. El-Serag HB, Mason AC. Rising incidence of hepatocellular carcinoma in the United States. N Engl J Med. 1999;340:745 750.

4. Deuffic $S$, Poynard T, Buffat $L$, et al. Trends in primary liver cancer. Lancet 1998;351:214-215.

5. Taylor-Robinson SD, Foster GR, Arora S, et al. Increase in primary liver cancer in the UK. Lancet 1997; 350:1 142-1143.

6. Bralet MP, Regimbeau JM, Pineau P, Dubois $S$, et al. Hepatocellular carcinoma occuring in non-fibrotic liver: epidemiologic and histopathologic analysis of 80 french cases. Hepatology 2000;32:200-204.

7. Nagasue N, Yukaya H, Hamada T, et al. The natural history of hepatocellular carcinoma. A study of 100 untreated cases. Cancer 1984;54:1461-1465.

8. The Liver Cancer Study Group of Japan. Predictive factors for long term prognosis after partial hepatectomy for patients with hepatocellular carcinoma in Japan. Cancer 1994;74:27722780.

9. Fong $Y$, Sun RL, Jarnagin $W$, et al. An analysis of 412 cases of hepatocellular carcinoma at a Western center. Ann Surg 1999;229:790-799

10. Esnaola NF, Mirza M, Lauwers GY, et al. Comparison of clinicopathologic characteristics and outcomes after resection in patients with hepatocellular carcinoma treated in the United States, France, and Japan. Ann Surg 2003;238:711-719.

11. Bismuth $H$, Chiche $L$, Adam $R$, et al. Liver resction versus transplantation for hepatocellular carcinoma in cirrhotic patients. Ann Surg 1993; 218:145-151.

12. Iwatsuki S, Starlz TE, Sheahan DG, et al. Hepatic resection versus transplantation for hepatocellular carcinoma. Ann Surg $1991 ; 214: 221-228$.

13. Livraghi T, Giorgio A, Marin G, Salmi A, et al. Hepatocellular carcinoma and cirrhosis in 746 patients : long-term results of percutaneous ethanol inyection. Radiology 1995;197:101-108.
14. Livraghi T, Goldberg SN, Lazzaroni S, Meloni F, et al. Hepatocellular carcinoma : redio-frecuency ablation of medium and large lesiones. Radiology 2000;214:761-768.

15. Llovet JM, Bru C, Bruix J. Prognosis of hepatocellular carcinoma: the BCLC staging classification. Sem Liver Dis 1999; 19:329-338.

16. Freeman RB. Liver allocation for HCC: A moving target. Liver Transpl 2004;10:49-51

17. Cha Ch, Fong $Y$, Jarnagin WR, Blumgart $L H$, et al. Predictors and patterns of recurrence after resection of hepatocellular carcinoma. J Am Coll Surg 2003;197:753-758.

18. Llovet JM, Fuster J, Bruix J. Intention to treat analysis of surgical treatment for early hepatocellular carcinoma: Resection versus transplantation. Hepatology 1999;30:1434-1440.

19. Wiesner RH, Edwards EB, Freeman RB, Harper A, et al. Model for end stage liver disease (MELD) and allocation of donor livers. Gastroenterology 2003;124:91-96.

20. Bruix J, Castells A, Bosch J, Feu F, et al. Surgical resection of hepatocellular carcinoma in cirrhotic patients : prognostic value of preoperative portal presure. Gastroenterology 1996; 111:1018-1022.

21. Cha Ch H, Ruo L, Fong Y, Jarnagin WR, et al. Resection of hepatocellular carcinoma in patients otherwise elegible for transplantation. Ann Surg 2003;238:315-323.

22. Figueras J, Jaurrieta $E$, Valls $C$, Ramos $E$, et al. Resection or transplantation for hepatocellular carcinoma in cirrhotic patients: outcomes based on indicated treatment strategy. J Am Collv Surg 2000;190:580-587.

23. Poon RT, Fan ST, Lo $C M$, et al. Long-term survival and pattern of recurrence after resection of small hepatocellular carcinoma in patients with preserved liver function: implications for a strategy of salvage transplantation. Ann Surg 2002;235:373-382.

24. Belghiti J, Cortes A, Abdalla EK, Régimbeau JM, et al. Resection prior to liver transplantation for hepatocellular carcinoma. Ann Surg 2003;238:885-893.

25. Adam R, Azoulay D, Castaing D, Eshkenazy R, et al. Liver resection as a bridge to transplantation for hepatocellular carcinoma on Cirrhosis. A reasonable strategy? Ann Surg 2003;238:508-519.

26. Adam R, McMaster P, O'Grady JG, Castaing D, et al. Evolution of liver transplantation in Europe: Report of the European Liver Transplant Registry. Liver Transpl 2003;9:1231-1243.

27. Mazzafero V, Regalia E, Doci R, Andreola S, et al. Liver transplantation for the treatment of small hepatocellular carcinoma in patients with cirrhosis. N Engl J Med 1996;334:693-699.

28. Adam R, del Gaudio M. Evolution of liver transplantation for hepatocellular carcinoma. J Hepatol 2003;39:888-895. 